\title{
SOLUTION OF A DIFFERENTIAL EQUATION AND ITS APPLICATIONS ${ }^{* \dagger}$
}

\author{
LIAN-ZHONG YANG
}

\begin{abstract}
In this paper, we proved that the order of entire solutions of the differential equation $f^{(n)}-f e^{Q(z)}=1$ are infinite, where $Q(z)$ is a nonconstant polynomial, and gave some of its applications.
\end{abstract}

\section{Introduction}

In this paper a meromorphic function will mean meromorphic in the whole complex plane. We say that two meromorphic functions $f$ and $g$ share a finite value $a$ IM (ignoring multiplicities) when $f-a$ and $g-a$ have the same zeros. If $f-a$ and $g-a$ have the same zeros with the same multiplicities, then we say that $f$ and $g$ share the value $a \mathrm{CM}$ (counting multiplicities). It is assumed that the reader is familiar with the standard symbols and fundamental results of Nevanlinna theory, as found in [3], [7].

L. Rubel and C. C. Yang proved the following result.

THEOREM A ([6]). Let $f$ be a nonconstant entire function. If $f$ and $f^{\prime}$ share two finite, distinct values $C M$, then $f \equiv f^{\prime}$.

Regarding Theorem A, a natural question is:

QUESTION 1. What can be said when a nonconstant entire function $f$ shares one finite value $\mathrm{CM}$ with one of its derivatives $f^{(k)}(k \geq 1)$ ?

We mention several results that concern with the Question 1.

THEOREM B ([4]). Let $f$ be a nonconstant meromorphic function, and let $a \neq 0$ be a finite constant. If $f, f^{\prime}$, and $f^{\prime \prime}$ share the value a $C M$, then $f \equiv f^{\prime}$.

\footnotetext{
*1991 Mathematics Subject Classification: 30D35, 30D20.

${ }^{\dagger}$ Key words: entıre functions, shared value, uniqueness theorem.

$\ddagger$ This work was supported by the NSF of China.

Received February 5, 1999.
} 
THEOREM C ([4]). Let $f$ be a nonconstant entire function, and let $a \neq 0$ be $a$ finite constant. If $f$ and $f^{\prime}$ share the value a IM, and if $f^{\prime \prime}(z)=a$ whenever $f(z)=a$, then $f \equiv f^{\prime}$.

THEOREM D ([9]). Let $f$ be a nonconstant entire function, let $a \neq 0$ be a finite constant, and let $n$ be a positive integer. If $f$ and $f^{\prime}$ share the value $a C M$, and if $f^{(n)}(z)=f^{(n+1)}(z)=a$ whenever $f(z)=a$, then $f \equiv f^{(n)}$.

THEOREM E ([1]). Let $f$ be an entire function which is not constant. If $f$ and $f^{\prime}$ share the value $1 C M$, and if $N\left(r, 0, f^{\prime}\right)=S(r, f)$, then

$$
\frac{f^{\prime}-1}{f-1}=c
$$

where $c$ is a non-zero constant.

THEOREM F ([8]). Let $f$ be a non-constant meromorphic function. If $f$ and $f^{\prime}$ share the value $1 C M$, and if

$$
\bar{N}(r, f)+N\left(r, \frac{1}{f^{\prime}}\right)<(\lambda+o(1)) T\left(r, f^{\prime}\right)
$$

for some real constant $\lambda \in(0,1 / 2)$, then $f$ and $f^{\prime}$ satisfy

$$
\frac{f^{\prime}-1}{f-1}=c \text {. }
$$

THEOREM G ([8, Corollary 3]). Let $f$ be a non-constant entire function, $k$ be a positive integer. If $f$ and $f^{(k)}$ share the value $1 C M$, and if

$$
\bar{N}\left(r, \frac{1}{f^{\prime}}\right)<(\lambda+o(1)) T(r, f)
$$

for some real constant $\lambda \in(0,1 / 4)$, then

$$
\frac{f^{(k)}-1}{f-1}=c
$$

for some non-zero constant $c$.

By considering solutions of the following differential equation

$$
\frac{f^{(k)}-1}{f-1}=e^{z}
$$

we know, in general, that one can not get

$$
\frac{f^{(k)}-1}{f-1}=\text { const. }
$$

under the condition of Question 1, for entire functions of infinite order. 
In this paper, we will answer Question 1 and give some improvements of the above Theorems $\mathrm{A}-\mathrm{G}$ for entire functions of finite order.

\section{Lemmas}

LEMMA 1 ([2]). Let $F$ be a nonconstant meromorphic function of finite order $\rho$, and let $\varepsilon>0$ be a given constant, $k$ be a positive integer. Then there exists a set $E \subset[0,2 \pi)$ that has linear measure zero, such that if $\psi_{0} \in[0,2 \pi)-E$, then there is a constant $R_{0}=R_{0}\left(\psi_{0}\right)>0$ such that for all $z$ satisfying $\arg z=\psi_{0}$ and $|z| \geq R_{0}$, we have

$$
\left|\frac{F^{(k)}(z)}{F(z)}\right| \leq|z|^{k(\rho-1+\varepsilon)}
$$

LEMMA 2. Let $F$ be an entire function, and suppose that $\left|F^{(k)}(z)\right|$ is unbounded on some ray $\arg z=\phi$. Then there exists an infinite sequence of points $z_{n}=r_{n} e^{\imath \phi}$ where $r_{n} \rightarrow+\infty$, such that $F^{(k)}\left(z_{n}\right) \rightarrow \infty$ and

$$
\left|\frac{F\left(z_{n}\right)}{F^{(k)}\left(z_{n}\right)}\right| \leq(1+o(1))\left|z_{n}\right|^{k}
$$

as $z_{n} \rightarrow \infty$

Proof. Let

$$
M\left(r, F^{(k)}, \phi\right)=\max \left\{\left|F^{(k)}(z)\right|: 0 \leq|z| \leq r, \arg z=\phi\right\} .
$$

Since $\left|F^{(k)}(z)\right|$ is unbounded on the ray $\arg z=\phi$, then there exists an infinite sequence of points $z_{n}=r_{n} e^{l \phi}$ where $r_{n} \rightarrow+\infty$, such that $F^{(k)}\left(z_{n}\right) \rightarrow \infty$ as $z_{n} \rightarrow \infty$ and

$$
M\left(r_{n}, F^{(k)}, \phi\right)=\left|F^{(k)}\left(z_{n}\right)\right|
$$

From

$$
F^{(k-1)}\left(z_{n}\right)=F^{(k-1)}(0)+\int_{0}^{z_{n}} F^{(k)}(u) d u
$$

we have

$$
\left|F^{(k-1)}\left(z_{n}\right)\right| \leq\left|F^{(k-1)}(0)\right|+\left|z_{n}\right|\left|F^{(k)}\left(z_{n}\right)\right| .
$$

Again, by

$$
\begin{aligned}
F^{(k-2)}\left(z_{n}\right) & =F^{(k-2)}(0)+\int_{0}^{z_{n}} F^{(k-1)}(u) d u \\
& =F^{(k-2)}(0)+\int_{0}^{z_{n}}\left\{F^{(k-1)}(0)+\int_{0}^{u} F^{(k)}(s) d s\right\} d u,
\end{aligned}
$$


we obtain

$$
\left|F^{(k-2)}\left(z_{n}\right)\right| \leq\left|F^{(k-2)}(0)\right|+\left|F^{(k-1)}(0)\right|\left|z_{n}\right|+\left|F^{(k)}\left(z_{n}\right)\right|\left|z_{n}\right|^{2} .
$$

By using the same methods for $n$ time, we deduce that

$$
\begin{aligned}
\left|F\left(z_{n}\right)\right| & \leq|F(0)|+\left|F^{\prime}(0)\right|\left|z_{n}\right|+\cdots+\left|F^{(k)}\left(z_{n}\right)\right|\left|z_{n}\right|^{k} \\
& =(1+o(1))\left|F^{(k)}\left(z_{n}\right)\right|\left|z_{n}\right|^{k}
\end{aligned}
$$

and we obtain

$$
\left|\frac{F\left(z_{n}\right)}{F^{(k)}\left(z_{n}\right)}\right| \leq(1+o(1))\left|z_{n}\right|^{k}
$$

as $z_{n} \rightarrow \infty$.

\section{Solutions of a differential equation}

THEOREM 1. Let $Q(z)$ be a nonconstant polynomial and $k$ be a positive integer. Then every solution $F$ of the differential equation

$$
F^{(k)}-e^{Q(z)} F=1
$$

is an entire function of infinite order.

Proof. It is well known that every solution of equation (1) is entire. We prove Theorem 1 by contradiction. Assume that Theorem 1 is not true, i.e., suppose that $F$ is a solution of equation (1) that has finite order $\rho$.

From (1),

$$
\frac{F^{(k)}}{F}-e^{Q(z)}=\frac{1}{F} .
$$

Let $\varepsilon>0$ be any given constant. Then from Lemma 1, there exists a set $E \subset[0,2 \pi)$ that has linear measure zero, such that if $\psi_{0} \in[0,2 \pi)-E$, then there is a constant $R_{0}=R_{0}\left(\psi_{0}\right)>0$ such that for all $z$ satisfying $\arg z=\psi_{0}$ and $|z| \geq R_{0}$, we have

$$
\left|\frac{F^{(k)}(z)}{F(z)}\right| \leq|z|^{k(\rho-1+\varepsilon)} .
$$

Now suppose that $\theta$ is any real number that satisfies $\theta \in[0,2 \pi)-E$, and for every $\alpha>0$,

$$
\frac{\left|e^{Q\left(r^{\ell \theta}\right)}\right|}{r^{\alpha}} \rightarrow+\infty
$$

as $r \rightarrow+\infty$. Then from (4), (3), and (2), it follows that 


$$
F\left(r e^{i \theta}\right) \rightarrow 0
$$

as $r \rightarrow+\infty$.

Now suppose that $\phi$ is any real number that satisfies $\phi \in[0,2 \pi)$, and for every $\beta>0$,

$$
r^{\beta} e^{Q\left(r e^{\imath \phi}\right)} \rightarrow 0
$$

as $r \rightarrow+\infty$. We now show that $\left|F^{(k)}(z)\right|$ is bounded on the $\operatorname{ray} \arg z=\phi$. Assume the contrary, i.e., suppose that $\left|F^{(k)}(z)\right|$ is not bounded on the ray $\arg z=\phi$. Then from Lemma 2, there exists an infinite sequence of points $z_{n}=r_{n} e^{l \phi}$ where $r_{n} \rightarrow+\infty$, such that $F^{(k)}\left(z_{n}\right) \rightarrow \infty$ and

$$
\left|\frac{F\left(z_{n}\right)}{F^{(k)}\left(z_{n}\right)}\right| \leq(1+o(1))\left|z_{n}\right|^{k}
$$

as $z_{n} \rightarrow \infty$. Since $F^{(k)}\left(z_{n}\right) \rightarrow \infty$, it follows from (6) and (1) that $F\left(z_{n}\right) \rightarrow \infty$. Then from (7), (6), and (2), we obtain that $F^{(k)}\left(z_{n}\right) \rightarrow 1$, which contradicts $F^{(k)}\left(z_{n}\right) \rightarrow \infty$. This contradiction proves that $\left|F^{(k)}(z)\right|$ must be bounded on the ray $\arg z=\phi$. By considering the formula

$$
F^{(k-1)}(z)=F^{(k-1)}(0)+\int_{0}^{z} F^{(k)}(w) d w
$$

we obtain that

$$
\left|F^{(k-1)}(z)\right| \leq\left|F^{(k-1)}(0)\right|+M|z|
$$

for all $z$ satisfying $\arg z=\phi$, where $M=M(\phi)>0$ is some constant.

Similarly, by using (8) and the formula

$$
F^{(J-1)}(z)=F^{(J-1)}(0)+\int_{0}^{z} F^{(j)}(w) d w, \quad(1 \leq j \leq k)
$$

for $k$ times, we can easily obtain

$$
|F(z)| \leq p_{k}(|z|)=(M+o(1))|z|^{k}, \quad \text { as } r \rightarrow \infty
$$

for all $z$ satisfying $\arg z=\phi$, where $p_{k}(x)$ is a polynomial of degree $k$.

We have shown that (9) holds for any $\phi \in[0,2 \pi)$ with the property (6), and that (5) holds for any $\theta \in[0,2 \pi)-E$ with the property (4). Since $Q(z)$ is a nonconstant polynomial, there exist only finitely many real numbers in $[0,2 \pi)$ that do not satisfy either (6) or (4). We also note that the set $E$ has linear measure zero. Therefore, since $F$ has finite order, it can be deduced from (9), (5), the Phragmén-Lindelöf theorem [5, pp. 270-271], and Liouville's theorem, that $F$ must be a polynomial with $\operatorname{deg} F \leq k$. But this is impossible because $Q(z)$ is nonconstant in (1). This contradiction proves Theorem 1 . 


\section{Applications of Theorem 1}

THEOREM 2. Let $f$ be a nonconstant entire function of finite order, and let $a \neq 0$ be a finite constant. If $f, f^{(k)}$ share the value a $C M$, then

$$
\frac{f^{(k)}-a}{f-a}=c
$$

for some non-zero constant $c$.

Proof. Since $f$ has finite order, and since $f$ and $f^{(k)}$ share the value $a \mathrm{CM}$, it follows from the Hadamard factorization theorem that

$$
\frac{f^{(k)}-a}{f-a}=e^{Q(z)},
$$

where $Q(z)$ is a polynomial. Set $F=(f / a)-1$. Then from (11),

$$
F^{(k)}-e^{Q(z)} F=1 .
$$

If $Q(z)$ is nonconstant, then from (12) and Theorem 1, we obtain that $F$ has infinite order. Since $f$ has finite order, this is impossible. Hence $Q(z)$ is a constant. Then from (11), we obtain (10) for a non-zero constant $c$.

Obviously, Theorem 2 improves Theorem E, Theorem F and Theorem G for entire functions of finite order. The following theorems follow from Theorem 2, which are the improvements of Theorems A, B, C and D.

THEOREM 3. Let $f$ be a nonconstant entire function of finite order, and let $a \neq 0$ be a finite constant, $k$ be a positive integer. If $f$ and $f^{(k)}$ share the value $a$ $C M$, and if there exists one point $z_{0}$ such that $f^{(k)}\left(z_{0}\right)=f\left(z_{0}\right) \neq a$, then $f \equiv f^{(k)}$.

COROLlaRY. Let $f$ be a nonconstant entire function of finite order, $k$ be $a$ positive integer, and let $a \neq 0, b$ be two distinct fintte values. If $f$ and $f^{(k)}$ share the value a CM, $f$ and $f^{(k)}$ share the value $b I M$, then $f \equiv f^{(k)}$.

THEOREM 4. Let $f$ be a nonconstant entire function of finite order, let $a \neq 0$ be a finite constant, and let $n$ be a positive integer. If $f$ and $f^{\prime}$ share the value a $C M$, and if there exists at least one point $z_{0}$ such that $f^{(n)}\left(z_{0}\right)=f^{(n+1)}\left(z_{0}\right) \neq 0$, then $f \equiv f^{\prime}$.

THEOREM 5. Let $f$ be a nonconstant entire function of finite order, let $a \neq 0$ be a finite constant, and let $n$ be a positive integer. If $f$ and $f^{(n)}$ share the value $a$ $C M$, and if there exists at least one point $z_{0}$ such that $f^{\prime}\left(z_{0}\right)=f^{(n+1)}\left(z_{0}\right) \neq 0$, then $f \equiv f^{(n)}$. 
Acknowledgement. The author would like to thank Professor Hong-Xun Yi for valuable discussions concerning this paper.

\section{REFERENCES}

[1] R. BRÜCK, On entıre functıons which share one value CM with their first derıvatıve, Results Math., 30 (1996), 21-24.

[2] G. GunderSEN, Estımates for the logarithmic derivative of a meromorphic function, plus similar estımates, J. London Math. Soc. (2), 37 (1988), 88-104.

[3] W K. Hayman, Meromorphic Functions, Clarendon Press, Oxford, 1964.

[4] G. Jank, E. Mues, and L. VolkmanN, Meromorphe Funktionen, die mit ihrer ersten und zweiten Ableitung einen endlichen Wert teilen, Complex Variables Theory Appl., 6 (1986), $51-71$.

[5] A. Markushevich, Theory of Functions of a Complex Variable, Volume 2, translated by R. Silverman, Prenctice-Hall, Englewood Cliffs, 1965.

[6] L. Rubel and C. C. Yang, Values shared by an entıre function and its derivative, Complex Analysis, Lecture Notes in Math., 599, Springer-Verlag, Berlin, 1977, 101-103.

[7] H. X. Yi AND C. C. YANG, Uniqueness Theory of Meromorphic Functions, Science Press, Beijing, 1995, in Chinese.

[ 8 ] Q. C. ZhANG, The uniqueness of meromorphic functions with their derivatives, Kodai Math. J., 21 (1998), 179-184.

[9] H. Zhong, Entire functions that share one value with their derivatives, Kodai Math. J., 18 (1995), 250-259.

Department of Mathematics

SHANDONG UNIVERSITY

JinAN, SHANDONG, 250100

P.R. CHINA

E-mail: lzyang@sdu.edu.cn 